\title{
FETAL FIBRONECTIN FFN. BIOCHEMICAL MARKERS OF PRETERM BIRTH
}

\author{
Nikolai Kolev, Stephan Ivanov, Emil Kovachev, Stanislav Slavchev \\ Department of Obstetrics and Gynecology, Medical University of Varna, Bulgaria
}

\begin{abstract}
The use of biochemical markers for predicting preterm birth has a potential advantage because it provides direct evidence of changes in the extracellular matrix of the surface between fetal membranes and the decidual tissue $(1,12)$. Fetal fibronectin ( $\mathrm{fFN})$ is a protein that is produced during pregnancy and acts as a biological glue which keeps the amniotic sac attached to the endometrium. fFN can be found in cervico-vaginal secretions up to 22 weeks and later in the last trimester (7). The purpose of this study is to determine the level of fFN in cervical mucus as a specific indicator of preterm birth in pregnant women with clinical symptoms. The study was attended by 90 women divided into two groups. The first group of pregnant women were at term gestation 24-34 weeks with clinical symptoms of PB and the second group - with normal pregnancy. Full Term Test was conducted in all women. The results were statistically processed using SPSS v.17. The presence of symptoms of preterm labor showed difference in the percentage of positive results of the fFN test $(p<0.05)$, women with clinical symptoms had a higher percentage of positive tests. When conducting the Full Term pregnancy test with positive results in the highest percentage were women with overt clinical PB, Roma and those with second and third birth. Furthermore, for pregnant women with a positive test result with children mainly born at 35 weeks, the newborns weighed an average of $2,550.1 \mathrm{~g}$, which explicitly includes them in the premature population.
\end{abstract}

Keywords: fFN, preterm birth, biochemical marker, Full Term test, risk factors, a positive result

\section{INTRODUCTION}

fFN is a high-molecular adhesive extracellular matrix glycoprotein. In the embryonic period, it is produced by the cells of cytotrophoblast (2). fFN is produced during pregnancy and acts as a biological glue by holding the amniotic sac attached to the endometrium (uterine lining). fFN can be found in

\author{
Address for corespondence \\ Nikolai Kolev \\ Department of Obstetrics and Gynecology, Medical \\ University of Varna \\ 55 M. Drinov Str. \\ 9000, Varna, Bulgaria
}

Received: November 18, 2013

Accepted: December 18, 2013 cervico-vaginal secretions up to 22 weeks and later at the end of the last trimester ( 1 to 3 weeks before birth). fFN is usually missing in the mucus between 24 and 34 weeks (5 $1 / 2$ to $81 \frac{1}{2}$ months) $(3,4,10)$.

The test usually takes place between 24 and 34 weeks. The presence of fFN during these weeks, along with symptoms of labor, suggests that the "glue" may decay and there is early detection of possible preterm labor $(8,11)$.

The presence of $f F N$ in the vagina of a pregnant woman up to 22 weeks is normal. In a normal pregnancy, between 22 weeks and 35 weeks, fFN should not be found in the cervico-vaginal secretions (6). Its presence is a highly informative biological marker for a risk of PB. Monoclonal antibody FDC- 6 was used in the test, which specifically recognizes the III-CS region of the fetal isoform of fibronectin $(5,9)$. 
The purpose of this study is to determine the level of fFN in cervical mucus as a specific indicator of $\mathrm{PB}$ in pregnant women with clinical symptoms.

\section{MATERIAL AND METHODS}

In a prospective study conducted in the maternity ward of the University Hospital „St. Anna“Varna in the period August 2012 - August 2013, two main groups of pregnant women were included. The 1-st group included 60 women with a pregnancy term 24-34 weeks and clinical symptoms of PB. The 2-nd (control) group included 30 pregnant women with normal (physiologically) ongoing pregnancy. Both groups took the Full Term test. The test detects levels of fFN $\geq 0.050 \mu \mathrm{g} / \mathrm{mL}$. The result are ready in 10 minutes, including the taking of secretion. Evidence of a negative test result: absence of fFN in women with no symptoms gives a $99 \%$ confidence that in the next 10-14 days PB will start; only $1 \%$ of women with negative results between 22 weeks and 24 weeks will give birth before 28 weeks. The negative result helps reduce unnecessary hospitalization and unnecessary use of medication. Indications of a positive test result: pregnant women with signs of labor and a positive test have a $40 \%$ chance to give birth prematurely; pregnant women without signs of labor and a positive test between weeks 22 and 24 weeks are 60 times more likely to give birth in the next 4 weeks. The positive result allows the doctor to take steps to delay the birth, as well as to use corticosteroids for lung maturation and thus to improve the outcome of $\mathrm{PB}$ and reduce health risks to infants.

To process the results the statistical program SPSS v. 17 was used. Dispersion, correlation and comparative analysis was applied to prove the intended objective.

\section{RESULTS}

The results of the test for fFN from 90 mothers were positive for $33.30 \%$, meaning high risk of $\mathrm{PB}$ (Fig. 1).

The presence of symptoms of preterm labor showed difference in the percentage of positive results of the fFN test $(\mathrm{p}<0.05)$ and women with clinical symptoms had a higher percentage of positive tests.

In terms of ethnicity, we can say that with the exception of Roma ethnicity, where positive tests constitute approximately half of the group, in other mothers the percentage of positive tests is approximately one quarter of the respective group (Fig. 2).

With regard to the number of births and fFN test results we can say that it keeps the same pattern as in pregnancy. Again, approximately $1 / 4$ of mothers with a first and third birth give positive test results, while mothers, who give birth to a second child have positive results in one third of cases (Fig. 3).

From the viewpoint of the gestational age of the infant there was a significant difference between

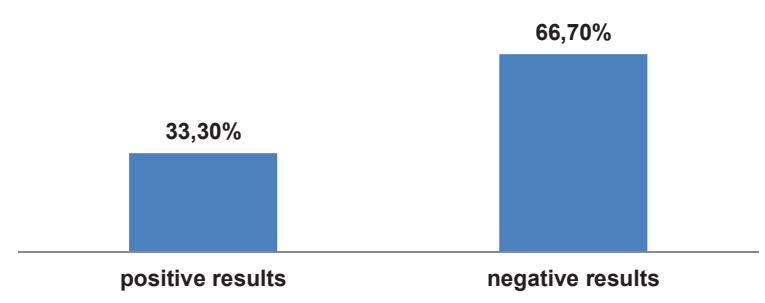

Fig. 1. Result of Full term test

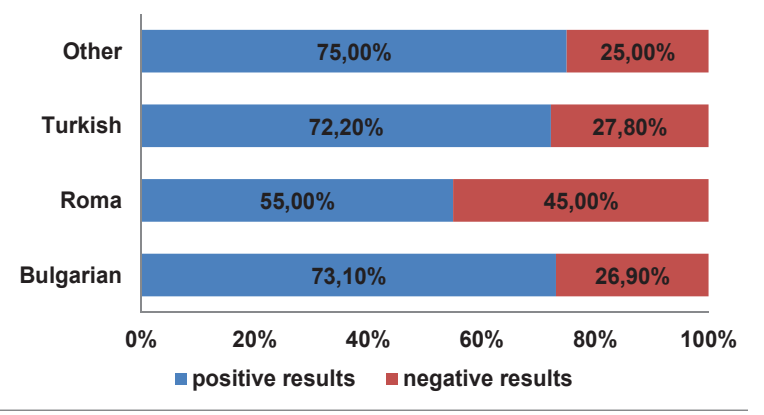

Fig. 2. Results of Full term test according to the ethnicity of the mothers

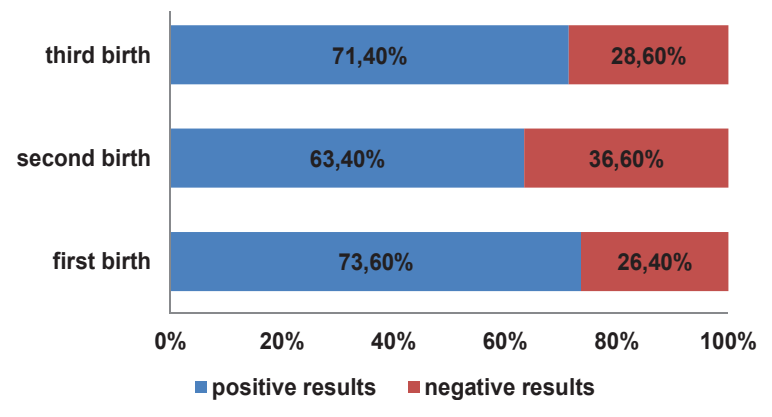

Fig. 3. Results of Full term test in the order of birth of mothers 
the mean age of the negative and positive results ( $\mathrm{p}$ $<0.001)$ and the age of the newborns with a negative result is higher than that of the ones with a positive result (Fig. 4).

The weight of the newborns also showed a significant difference in the two results of the fFN test ( $p<0.001)$, babies with positive results having a relatively lower weight than the infants with normal birth (Fig. 5).

It was proven by correlation analysis that there is an extremely strong proportional correlation $(\mathrm{r}$ $=0,97 ; \mathrm{p}<0,001)$ between the gestational age of the fetus and its weight in women who tested positive in the fFN test.

\section{DISCUSSION}

There was no significant difference in the number of births for the positive results from the test between women with and without clinical symptoms at first birth. The latter showed approximately 2 -fold

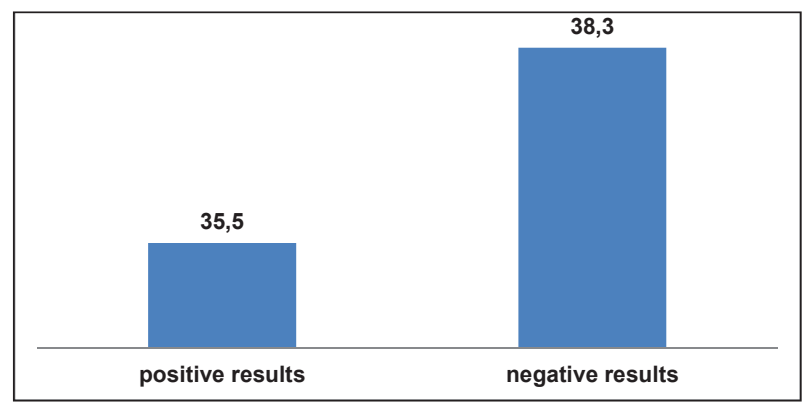

Fig. 4. Results of Full term test and an average gestational age of the newborn

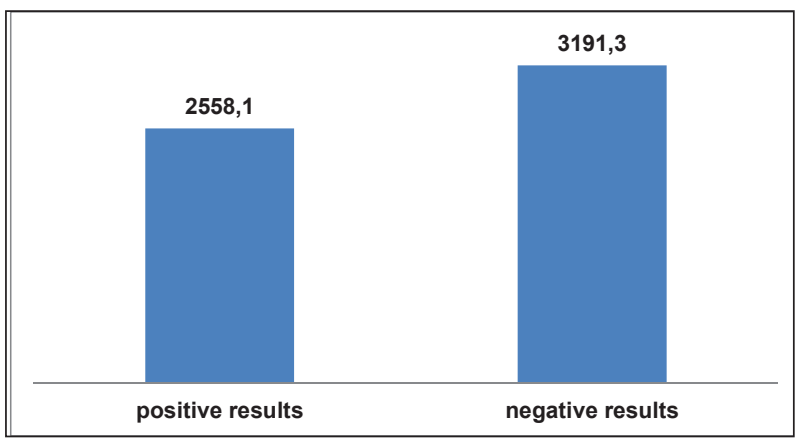

Fig. 5. Results of Full term test and the average birth weight lower levels of positive tests at first birth compared to the clinical pregnancy group $(\mathrm{p}<0.01)$.

Birth weight in women with a negative result was approximately $400 \mathrm{~g}$ higher than that of children born to women with positive test results $(\mathrm{p}<0.001)$. However, children born to the second group of women were slightly above the weight limit for $\mathrm{PB}$ (about $2600 \mathrm{~g}$ ). For the first group of women, the infants had predominantly normal birth weight (average weight of $3200 \mathrm{~g}$ ).

Difference was also observed in the gestational age of the fetus $(p<0.001)$ - women with positive test results and presence of clinical symptoms gave birth 3 weeks earlier, which puts them in the group of PBs (average gestational age in women without symptoms was 38 weeks, and in women with symptoms - 35 weeks gestation).

\section{CONCLUSIONS}

In clinical practice we often encounter pregnant women with clinical symptoms of $\mathrm{PB}$ when you need to predict the onset of parturition and need assessment of the feasibility for the birth to be postponed or delayed until the most favorable gestational week for the survival of fetus.

The application of rapid immunoassay tests from 24 to 34 weeks, enables us for a very short period of time - 10 to 15 minutes, to define high-risk mothers and take the necessary therapeutic measures to prevent $\mathrm{PB}$.

After conducting a Full Term pregnancy test with positive results in the highest percentage were women with overt clinical PB, Roma and second and third birth women. Furthermore, pregnant women with a positive test result mainly gave birth at 35 weeks, with newborns weighing an average of 2,550.1 $\mathrm{g}$, which explicitly includes them in the premature birth population.

The Full Term test has high sensitivity and specificity, which gives us reason to believe that the resulting positive and negative values are sufficient grounds to accept or reject the risk of occurrence of PB. 


\section{REFERENCES}

1. ACOG news release October 31, 2003:

Progesterone recommended in certain high risk pregnancies to help prevent preterm birth.

2. American College of Obstetrics and Gynecologists (ACOG). Assessment of Risk Factors for Preterm Birth. ACOG Practice Bulletin, number 31, October 2001 (reaffirmed 2008).

3. Andersen HF. Use of fetal fibronectin in women at risk for preterm delivery. Clin Obstet Gynecol 2000; 43: 746-758.

4. Ascarelli MH, Morrison JC. Use of fetal fibronectin. Obstet Gynecol Surv 1997, 52: 1-12.

5. Bashore RA, Westlake JR. Plasma values of unconjugated estriol in high-risk pregnancy. Am J Obstet Gynecol 1997; 128: 371-80.

6. Berghella, V., et al. Fetal Fibronectin Testing for Reducing the Risk of Preterm Birth (Review). The Cochrane Library, 2009, Issue 2.

7. Goldenberg R.L., J.F. Culhane, J.D. Iams, et al. Epidemiology and causes of preterm birth. Lancet, 2008; 5; 371(9606): 75-84.

8. Honest H., L.M. Bachman, J.K. Kleijnen, K.S. Khan. Accuracy of cervico-vaginal fetal fibronectin test in predicting risk of spontaneous preterm birth: systematic review. BMJ, 2002; 325:301-320.

9. Malinova M., Clinical behavior cervix. Midwifery and gynecology. Obstet and Gynecol, 2013; 1:41-48.

10. Meis P., Klebanoff M., Thom E. et al. Prevention of recurrent preterm delivery by 17 alphahydroxyprogesterone caproat. NEJM, 2003; 348: 2379-2385.

11. The American College of Nurse-Midwives (ACNM). Division of Standards and Practice Clinical Standards and Documents Section. Prevention of Preterm Labor and Preterm Birth, June 2012.

12. Yast J.D., G. Lu. Biochemical markers for the prediction of preterm delivery. Clin Perinatol, 2007 Dec; 34(4):573-586. 